\title{
BMJ Open Benefits finding among Chinese family caregivers of stroke survivors: a qualitative descriptive study
}

\author{
Yong-Xia Mei (D) , ${ }^{1,2,3}$ Beilei Lin (D) , ${ }^{1}$ Weihong Zhang, ${ }^{1}$ Dong-Bin Yang, ${ }^{3}$ \\ Shan-Shan Wang, ${ }^{1,4}$ Zhen-Xiang Zhang, ${ }^{1}$ Daphne Sze Ki Cheung ${ }^{4}$
}

To cite: Mei Y-X, Lin B, Zhang W, et al. Benefits finding among Chinese family caregivers of stroke survivors: a qualitative descriptive study. BMJ Open 2020;10:e038344. doi:10.1136/ bmjopen-2020-038344

- Prepublication history for this paper is available online. To view these files, please visit the journal online (http://dx.doi. org/10.1136/bmjopen-2020 038344).

Received 10 March 2020 Revised 02 September 2020 Accepted 08 September 2020

A) Check for updates

(C) Author(s) (or their employer(s)) 2020. Re-use permitted under CC BY-NC. No commercial re-use. See rights and permissions. Published by BMJ.

${ }^{1}$ School of Nursing and Health, Zhengzhou University, Zhengzhou, China

${ }^{2}$ College of Public Health, Zhengzhou University, Zhengzhou, China

${ }^{3}$ Teaching office, The People's Hospital of Hebi, Hebi, China

${ }^{4}$ School of Nursing, The Hong Kong Polytechnic University, Hong Kong, China

Correspondence to Professor Zhen-Xiang Zhang; zhangzx6666@126.com, Daphne Sze Ki Cheung; daphne.cheung@polyu.edu. hk and

Professor Dong-Bin Yang; dongbinyang@126.com

\section{ABSTRACT}

Objectives The caregiving experience includes both caregiver burdens and benefits finding. However, the benefits obtained by family caregivers of stroke survivors in Chinese community dwellings are unknown. The objective of this study was to explore the benefits experienced by family caregivers of stroke survivors in Chinese community dwellings.

Design A qualitative descriptive design was used, fulfilling the consolidated criteria for the Standards for Reporting Qualitative Research reporting guidelines. Semi-structured interviews were conducted with 20 family caregivers of stroke survivors. The interviews were audiotaped, transcribed and analysed. Thematic analysis was performed to analyse the interview transcripts.

Setting and participants Home interviews were conducted with family caregivers of stroke survivors in two communities in Zhengzhou, China

Results The family caregivers of stroke survivors experienced various benefits from caregiving. There were both internal benefits (increases in knowledge and skills, the development of positive attitudes, and the development of a sense of worthiness and achievement) and external benefits (family growth and gains in social support), which interact to create a healthy lifestyle.

Conclusion Our findings provide a comprehensive perspective in understanding the benefits perceived by family caregivers of stroke survivors. This study provides insights into interventions focused on identifying benefits finding in six domains that may help reduce negative emotions and promote the mental health of caregivers.

\section{INTRODUCTION}

Stroke is the leading cause of disability in China. ${ }^{1}$ There are 130 million stroke survivors in China, and $60 \%$ of them are disabled and need continuous support from family members. ${ }^{2}$ Performing caregiving tasks can cause family caregivers to experience negative impacts such as emotional burden, anxiety and depression. ${ }^{3-5}$ Due to the traditional Chinese culture of respecting the elderly, survivors prefer to receive care and support from their spouses and children, who often lack professional caregiving skills. ${ }^{6}$ In addition, the Chinese government encourages

\section{Strengths and limitations of this study}

- Most studies focus on the negative aspects of stroke caregiving. A strength of this study is its focus on the perceived benefits of caregiving which have implications for rehabilitation research and practice.

- The use of semistructured interviews provided an opportunity to elicit detailed information about family caregivers' various benefits they perceived from caregiving.

- This study is limited by the lack of geographical diversity of the caregiver sample.

their people to take the initiative to care of their loved ones who need support. ${ }^{7}$ As a result, the caregivers are expected to provide more support, which places a heavy caregiving burden on them ${ }^{8}$; moreover, unlike in other developed countries, there is no government or community social service assistance, which leads to a greater burden on caregivers. ${ }^{9}$

Researchers have tried different ways to reduce these negative impacts, but the results have been critiqued as less than satisfactory. ${ }^{10}$ However, a high level of burden or anxiety and depression not only contributes to inadequacies in the physical and mental health of caregivers but also reduces the quality of the care provided to stroke survivors and the quality of life of both stroke survivors and caregivers. ${ }^{11-13}$ Focusing only on the negative part of caregiving may limit researchers and practitioners from understanding the holistic experience of caregivers.

However, family caregivers also experience benefits by performing caregiving tasks. Researchers have shown that caregivers may perceive benefits from caregiving, including creating a better relationship with stroke survivors, learning how to cope with stress and mastering new skills. ${ }^{14} 15$ Identifying the benefits perceived by caregivers not only contributes to better physical and mental health but also promotes quality of life. ${ }^{16}$ Previous 


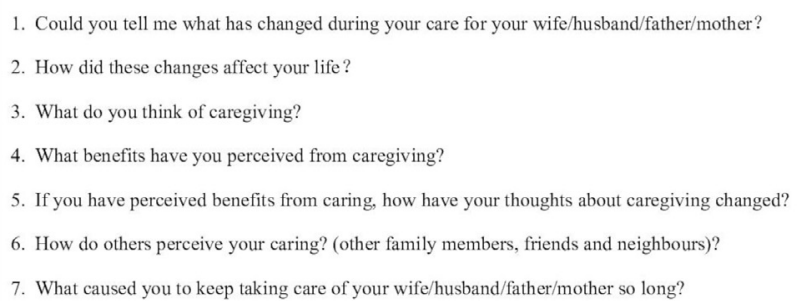

Figure 1 The written topic guide used in the interviews.

researchers have shown that the greater the level of benefit perceived by caregivers is, the lower the level of caregiver burden and depression; thus, identifying these benefits finding could play a moderating or mediating role in the relationship between caregiver burden and depression. ${ }^{18-20}$ In addition, the perception among caregivers of a higher level of benefits would also help care recipients. ${ }^{21}$ Hence, identifying these benefits finding may provide a new direction for providing psychological care for caregivers of stroke survivors from the positive view.

Different kinds of benefits perceived by caregivers have been reported in the literature, but those perceived by caregivers of stroke survivors have been less studied. An integrative review ${ }^{22}$ indicated that the benefits perceived by caregivers of patients with dementia included four domains, namely, a sense of personal accomplishment and gratification, feelings of mutuality in a dyadic relationship, and increases in family cohesion and functionality. An examination of the caregiving benefits perceived by the caregivers of patients with cancer suggested that they accepted that things could not be controlled, learnt effective skills to cope with stress, and found meaning in their own distress. ${ }^{23}$ Similarly, the caregivers of stroke survivors in Australia reported that they came to have better relationships with others and appreciated life more,${ }^{14}$ those in the USA developed a positive attitude towards the disease ${ }^{24}$ and those in the UK strengthened their sense of meaning and received compliments from care recipients. ${ }^{25}$

Chinese culture may have a significant impact on the caregiving experience, as caregivers see caring as natural due to filial piety. ${ }^{26}$ Adult Chinese children who take care of their parents are proud to be role models and set the standards of care for their next generation, and they possess a great sense of contentment thanks to the recognition of their family and society. ${ }^{27}$ Chinese caregivers of stroke survivors in hospitals located in Nanjing City, China, experienced reciprocal love and enhanced inner strength ${ }^{28}$; these findings may not capture the experiences of caregivers in other regions of China. Despite the increasing focus on this subject, research on the benefits perceived by caregivers of stroke survivors is still scant, ${ }^{29}$ especially for those who take care of their loved ones in a community-dwelling context common to traditional Chinese culture. Thus, this study aims to explore the benefits perceived by Chinese family caregivers of stroke survivors. The findings will provide insights to help professionals provide better support caregivers throughout their caregiving journey.

\section{METHODS}

Design

Qualitative and descriptive research was implemented to obtain first-hand information on the benefits that the family caregivers experienced. The consolidated criteria for the Standards for Reporting Qualitative Research were used to guide the reporting. ${ }^{30}$

\section{Sampling and participants}

Caregivers of stroke survivors discharged from two communities in Zhengzhou, China, at least 3 months prior were invited to participate by purposive sampling. The inclusion criteria for caregivers were as follows: (1) was a primary family caregiver (non-professional and unpaid) for a stroke survivor aged 18 or older who had a formal diagnosis of cerebrovascular disease and had a functional disability (Barthel Index $<100),{ }^{31}$ (2) provided care for at least 4 hours each day in the most recent 4 weeks and (3) was able to communicate in Mandarin and willing to take part in the interviews.

\section{Patient and public involvement}

The caregivers were only invited to affirm that the interview transcripts reflected their feelings and experience after the data analysis, but they were not invited to comment on the study design or contribute to the writing or editing of this document for readability or accuracy.

\section{Data collection}

Individual interviews were performed between November 2015 and January 2016 in the caregivers' homes without the presence of the stroke survivor. The interviews were semi-structured, and a written topic guide (figure 1) was used by the primary investigator, who is a psychiatrist with rich experience in interviews in Mandarin. The interviews were audio-recorded with the consent of the participants. Data collection was stopped after 20 participants were interviewed, as data saturation was reached (the primary investigator perceived that the content of the interviews was becoming repetitive and that no new information was emerging when she conducted the interviews). ${ }^{32}$ Data were coded concurrently with study recruitment and data collection. Moreover, the interview transcripts were also checked by our research team to make sure that no new content was emerging. The characteristics of stroke survivors and their family caregivers were collected by a community nurse who worked together with us.

\section{Data analysis}

The interviews were transcribed verbatim within 24 hours of the interview. Thematic analysis was conducted to identify, analyse and report themes within the data, including data familiarisation, coding and the development of themes and subthemes. ${ }^{33}$ The first and second authors (Y-XM and BL) read the transcribed interviews 
independently and highlighted and coded the text; the other two authors (WZ and D-BY) compared the codes, and minor disagreements were resolved through discussion. The codes were read several times and compared with the text. We collated similar codes and sub-categories into themes. Our team continued integrating data until no new categories could be generated and until any disagreements concerning the categories were resolved. We also checked the sub-categories for accuracy. Although the interviews were conducted in Chinese, the quotes were confirmed by family caregivers and were translated into English by Y-XM and S-SW and checked for accuracy by research team discussion in the stage of dissemination of the findings. ${ }^{34}$

\section{Trust worthiness}

We considered the aspects of credibility, transferability, conformability, dependability, and authenticity. ${ }^{35}$ To ensure the credibility, we chose various family caregivers participants from different sociodemographic backgrounds (different age stages, education levels, relationships with patients, durations of care and so on). We determined that the content of the data covered all the significant variations. We ensured the transferability of the data through an accurate and rich description of the context and culture; we provided the characteristics of participants, data collection, the process of the analysis and sufficient quotes. To facilitate conformability, all of the authors were trained to be qualified at qualitative research. Moreover, a qualitative research expert with experience in stroke care was invited to provide comments on the data analysis. We facilitated the dependability of the data through the researchers' training in interview skills, a clear data analysis process and a constant comparative analysis. We ensured the authenticity by means of the process we used as a team to generate the codes and the data analysis.

\section{Findings}

A total of 20 caregivers participated in this study. They were aged between 35 and 79 years, with a mean age of 58.6 years. The detailed characteristics of caregivers and stroke survivors are presented in tables 1 and 2.

The audio-recorded interviews lasted between 30 and $60 \mathrm{~min}$, with an average of approximately $42 \mathrm{~min}$. Six main themes and 14 subthemes, shown in table 3, were identified from the analysis of the interviews. The following section presents the themes and subthemes and the related quotes. The symbol (P1-P20) indicates the interview from which the quotations originated.

\section{Increases in knowledge and skills}

Acquiring knowledge

All participants reported that they had acquired knowledge on stroke diseases and health.

I had read lots of books about stroke, and I knew the importance of how to prevent the recurrence since my father had a stroke. (P1)
Table 1 Characteristics of stroke survivors $(n=20)$

$\%$

\begin{tabular}{|crc|}
\hline Age (years) & & \\
$<60$ & 5 & 25.0 \\
$\geq 60$ to $<70$ & 4 & 20.0 \\
$\geq 70$ & 11 & 55.0 \\
Gender & & \\
$\quad$ Male & 12 & 60.0 \\
Female & 8 & 40.0 \\
\hline
\end{tabular}

Highest education level

$\begin{array}{lll}\text { Primary } & 7 & 35.0 \\ \text { Secondary } & 7 & 35.0 \\ \text { Tertiary } & 6 & 30.0 \\ \text { Duration of disease } & & \\ \leq 1 & 7 & 35.0 \\ >1 \text { to } \leq 3 & 6 & 30.0 \\ >3 & 7 & 35.0\end{array}$

Household income per month ( $¥)$

\begin{tabular}{lrr}
$\leq 2000$ & 4 & 20.0 \\
$>2000$ to $\leq 3000$ & 7 & 35.0 \\
$>3000$ & 9 & 45.0 \\
Type of stroke & & \\
$\quad$ Ischaemic & 10 & 50.0 \\
Haemorrhagic & 4 & 20.0 \\
Mixed & 6 & 30.0 \\
Stroke number & & \\
1 & 12 & 60.0 \\
2 & 3 & 15.0 \\
$\geq 3$ & 5 & 25.0 \\
Severity of disability & & \\
Slight dependence & 3 & 15.0 \\
Moderate dependence & 10 & 50.0 \\
Severe dependence & 7 & 35.0 \\
\hline
\end{tabular}

We were aware of the importance of having knowledge about how to eat, how to exercise, and how to sleep healthily. (P5)

\section{Mastering caring skills}

Most participants mentioned that they had mastered many skills with respect to taking care of stroke survivors.

I am good at changing his clothes and sheets easily; you know, everything has its own tactics. (P7)

You have no choice, but you have to solve all the caring tasks in a better way; that is why I can now transfer him from the bed to the chair easily. (P6) 


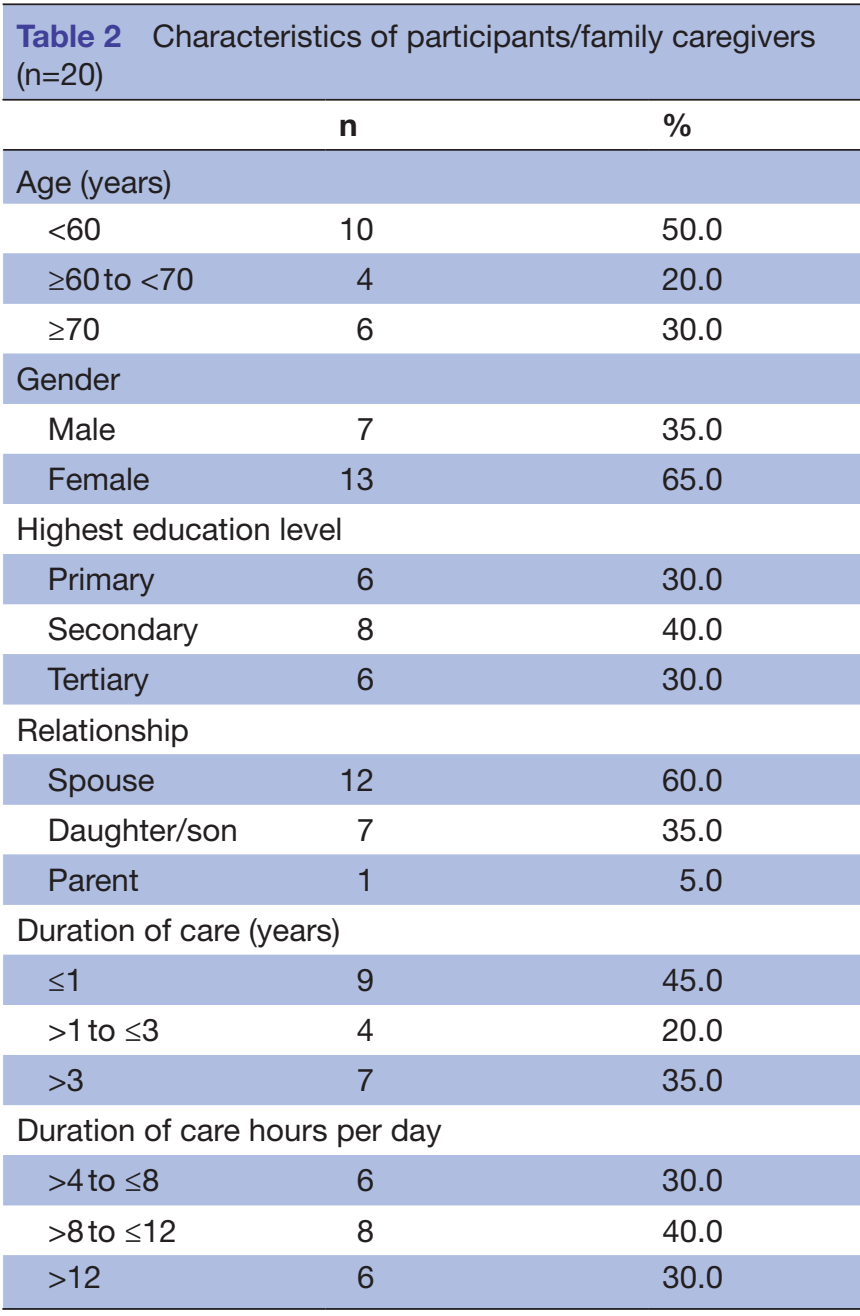

\begin{tabular}{|c|c|}
\hline Themes & Subthemes \\
\hline \multirow{4}{*}{$\begin{array}{l}\text { Increases in knowledge and } \\
\text { skills }\end{array}$} & Acquiring knowledge \\
\hline & Mastering caring skills \\
\hline & $\begin{array}{l}\text { Coping with stress much } \\
\text { more easily }\end{array}$ \\
\hline & $\begin{array}{l}\text { Being more careful and } \\
\text { cautious }\end{array}$ \\
\hline \multirow{4}{*}{$\begin{array}{l}\text { Development of positive } \\
\text { attitudes }\end{array}$} & Accepting reality \\
\hline & Cherishing what they have \\
\hline & Having a new goal to achieve \\
\hline & Being grateful \\
\hline \multirow{2}{*}{$\begin{array}{l}\text { Development of a sense of } \\
\text { worthiness and achievements }\end{array}$} & Worthiness \\
\hline & Achievements \\
\hline \multirow[t]{2}{*}{ Family growth } & $\begin{array}{l}\text { Having a more intimate } \\
\text { dyadic relationship }\end{array}$ \\
\hline & Growth of family members \\
\hline \multirow[t]{2}{*}{ Gains in social support } & Gains in friendships \\
\hline & Social benefits \\
\hline Taking up a healthy lifestyle & - \\
\hline
\end{tabular}

\section{Coping with stress much more easily}

Some participants noted that they could cope with stress more easily after providing and could handle the problems they faced when taking care of their loved ones.

I struggled for a long time, but now I am ready to risk everything; there is nothing that cannot be resolved. (P6)

I can't change anything, so I choose to face it; I like to sing when I am stressed. (P12)

\section{Being more careful and cautious}

Some participants revealed that caring for a stroke survivor made them more careful and cautious.

I am more careful than before; I check the medicine several time; I have to take care of everything. (P9)

I used to be careless, but after my mother was sick, I have to care about everything; I have to be careful. (P10)

\section{Development of positive attitudes \\ Accepting reality}

Some participants accepted the reality of the sickness of their loved ones, and they look at caregiving as a normal part of life now.

It is my turn now; I tried my best to take good care of her and accept the reality. (P9)

A fact is a fact. You have the option to live a life happily or sadly. Why not stay with your loved ones and live a happy life? (P20)

\section{Cherishing what they have}

Some participants stated that they began to cherish the people they love and the things that they do have.

We have each other, and we live together; that is what I cherish a lot. (P2)

When you go home, there is still someone you love waiting for you; this is home, so I treasure what I have. (P20)

Having a new goal to achieve

Some participants reported that their new goal was to take care of their loved ones as long as possible.

All I need to do is care for him well, and I believe he will get better with my help. (P17)

The biggest goal of my life now is taking good care of her. (P19)

\section{Being grateful}

Some participants expressed their thankfulness for their destiny, which allowed them the chance to take care of their loved one.

I am really grateful that I still have the chance to look after my mother and to do my filial duty. (P10) 
I want to thank destiny that we are still together; I am the lucky one compared to those who lost their loved ones. (P20)

\section{Development of a sense of worthiness and achievements Worthiness}

Some participants noted that they found value in being a caregiver.

Although I am old, I am still useful, and I am the pillar of our family. (P18)

I am willing to help new caregivers, and I am glad I have the ability to help. (P16)

Some participants perceived gratitude and praise from their loved ones and others.

He often holds my hands and smiles at me; I know he wants to thank me. (P12)

My relatives and neighbours respect me, and they think what I am doing is great. (P15)

\section{Achievements}

Some participants expressed that they perceived a sense of achievements from caring.

She is better and better. I perceive achievements. Every effort should be paid back. (P19)

You really have a sense of accomplishment when you see that she is well cared for. (P13)

\section{Family growth}

Having a more intimate dyadic relationship

Some participants reported that their relationships with their loved ones and their family members were better as a result of their caring.

I want to take care of her, stay with her and make her happy now. (P19)

Our family has become closer since he became sick, and our children often come back to visit us. (P7)

\section{Growth of family members}

Some participants noted that their family members had become more sensible and responsible.

My son became more sensible, and he would do some things for his grandfather; he really cares about his grandfather. (P1)

We count on each other; the sickness makes us grow, and we are becoming better people. (P18)

\section{Gains in social support}

Gains in friendships

Some participants stated that they made new friends and gained a great deal of support.

I know a friend whose father also had a stroke. We often call each other and have a walk together. We help each other. (P3)
A friend in need is a friend indeed; I really count on my friends now. (P4)

\section{Social benefits}

Some participants thought they had helped to perpetuate good traditions, which references good Chinese virtues, such as providing for the aged and respecting relatives. It is not only about their family, but also about the contribution they made to their society, and their society will support them.

My son respects me, and he learns from me. When I am old, I will need him just like my father needs me. I hope we can carry forward our filial piety; it is really great. (P11)

I do not want to bring burden to our country; this is my responsibility to take care of my mother. I want to become a model for my children. (P8)

\section{Taking up a healthy lifestyle}

Most participants stated that they not only developed good habits but also helped stroke survivors quit some bad habits; the participants and their care recipients both wanted to stay healthy.

We began to pay more attention to our health since she had a stroke. I gave up smoking and took her to have a walk nearly every day. (P14)

I supervised my mother's diet and exercise; also, for myself and other family members, health is the most important thing in our life, and we give priority to health. (P10)

Finally, we generated an affinity diagram, as shown in figure 2. Increases in knowledge and skills, the development of positive attitudes, and the development of a sense of worthiness and achievement are internal perceptions of caregivers; family growth and gains in social support are external perceptions of caregivers, which interact to promote the maintenance of healthy behaviour.

\section{DISCUSSION}

Although taking care of stroke survivors caused stress for the caregivers, all participants reported benefits and growth from caregiving.

Taking care of their loved ones brought caregivers not only practical benefits such as an increase in knowledge and skills but also the internal perception of benefits, for example, gaining the ability to cope with stress and a more careful and cautious character. Moreover, caregivers were able to accept reality, cherish everything they have and appreciate life. This finding confirms the work of previous researchers, ${ }^{36}$ who reported that caregivers were more patient, more sensitive to others' needs and more modest when communicating with others after beginning the caregiving experience. Our caregivers also confirmed the priorities in their lives and stated that they began to pay more attention to keeping in contact with others. 
Internal benefits

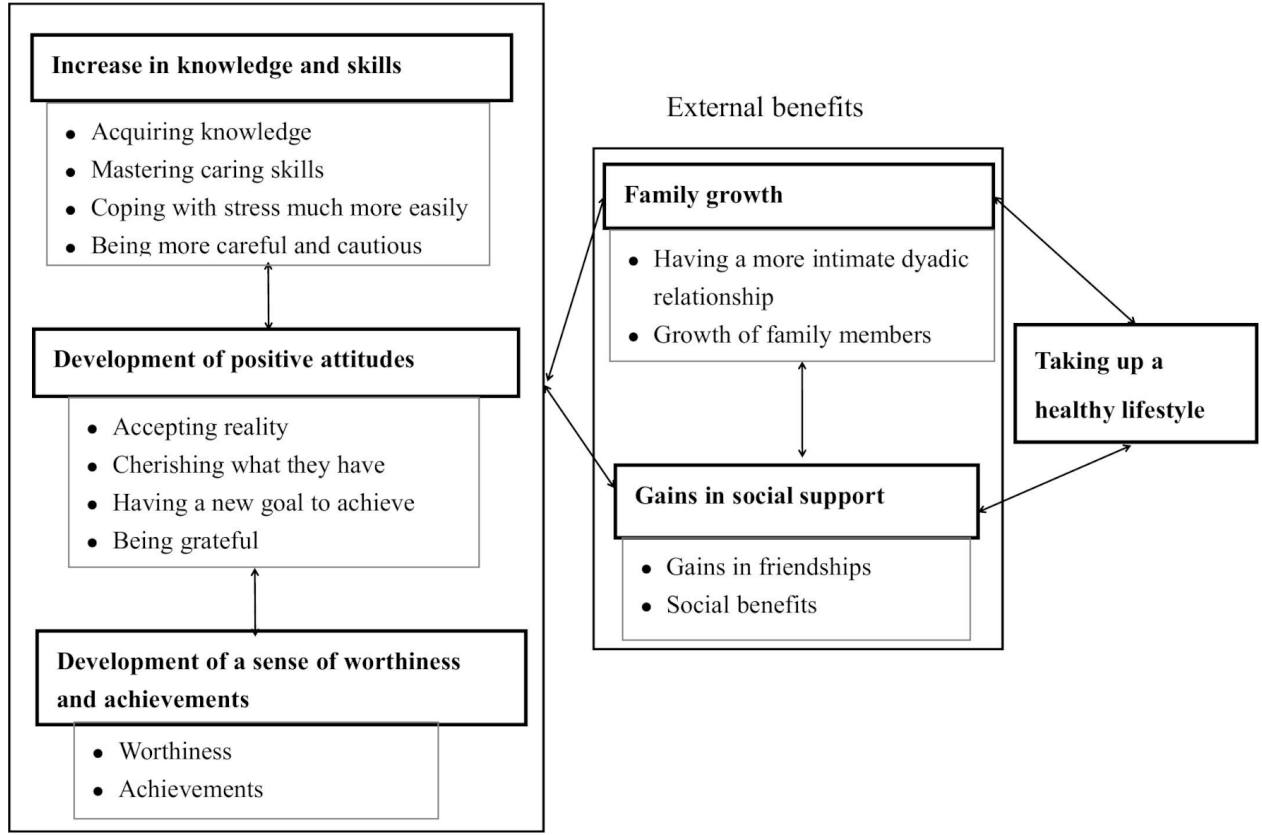

Figure 2 Affinity diagram showing six themes and subthemes concerning benefits finding of family caregivers of stroke survivors.

Thus, community nurses may try to help caregivers feel these benefits, which could help caregivers maintain a higher perception of these benefits.

Caregivers perceived values both internally (they felt more useful and sensed their achievements) and externally (they experienced gratitude from families and praise from relatives and neighbours). These values are important parts of benefit finding. ${ }^{22}$ Caregivers had to handle caring challenges and difficulties, which made them master new skills and strategies. ${ }^{37}$ Moreover, the improvements in the patients were also a source of achievement. ${ }^{38}$ Previous researchers have confirmed that more self-efficient skills help caregivers achieve more. ${ }^{39}$ Consequently, training caregivers to be capable of performing caregiving tasks and helping them to build their confidence can not only promote their benefit finding but also improve the quality of the rehabilitation of stroke survivors.

The relationship between caregivers and stroke survivors improved, and the entire family supported each other. Previous research has indicated that the benefits finding of caregivers and patients is mutual ${ }^{40}$ and that an improved relationship between caregivers and patients is an important benefit. ${ }^{22}$ One possible reason for the improved relationship may be that caregivers have enough time to communicate and talk with their loved ones. ${ }^{41}$ Sometimes caregivers thank their companions for the changes in their relationship. ${ }^{42}$ Another reason could be that caregivers want to care for their loved one as compensation. Support for the dyad process of psychological adaption in a well-timed manner after stroke should be focused on by rehabilitation interventions. ${ }^{43}$ As a result, the experience of caregivers (especially family-related benefits) provides a clue for designing interventions by regarding the family as a unit. ${ }^{44}$

Caregivers perceived social benefits they received through caregiving, including finding new friends and support from existing friends as well as continuing positive traditional family behaviours by acting as role models for their offspring. We also confirmed the findings of previous researchers that the caregivers improved their interpersonal relationships. ${ }^{15}$ Support can come from peers who have experienced similar situations and can be an essential social benefit and resource for caregivers. ${ }^{45}$ However, other researchers have reported that Chinese caregivers focus only on the care recipient and have limited time to make friends. ${ }^{46}$ In Chinese culture, caregivers think it is their duty and responsibility to take care of their family members, and they hope they will be treated as well by their children as they treat their parents. ${ }^{26}$ It is essential to remind Chinese caregivers to care about themselves, make friends, and find peer support for themselves. Professionals may provide more chances for caregivers to care for themselves or build a peer support group. Caregivers are essential in the rehabilitation of stroke survivors; therefore, encouraging caregivers to take care of themselves first and providing multi-level social support to caregivers are also indispensable. ${ }^{43}$

Our finding of six benefits domains provides theoretical and practical insights for the further study of the caregiver experience. Similar to other studies, our study is not without limitations. First, the participants were selfselected and came from a relatively affluent region of China; thus, their experiences may not reflect those of other caregivers in less affluent or rural areas in China. Second, the participants were limited to caregivers who 
were spouses, children or parents of stroke survivors; therefore, the finding may not reflect the perspectives of caregivers who are siblings or other relatives or caregivers from culturally and linguistically diverse backgrounds.

\section{CONCLUSION}

Our results add to the understanding of the benefits perceived by caregivers in community dwellings. The benefits and growth that come from caring include benefits for the caregivers themselves, benefits for families and gains in social support. These findings may provide new insights for professionals and suggest that future studies can focus on benefits rather than on the negative impact of caregiving. This finding also supports the assessment of caregivers and provides a framework for the development of a benefit-finding tool. Moreover, the study provides insights into benefit-focused interventions that may help promote the mental health of caregivers and stroke survivors.

Acknowledgements The authors thank all the caregivers of stroke survivors who shared their experience with us. We also want to thank Professor Xiaohong Liu for her selfless interview data consultation.

Contributors Y-XM designed and developed the study, analysed and interpreted the data, and drafted the initial manuscript. B-LL, D-BY and Z-XZ were involved in the development of the research question. Y-XM and S-SW were involved in the acquisition of data. B-LL, D-BY, WZ and DSKC assisted with analysis and interpretation of data. All the authors revised the drafts for intellectual content and edited the manuscript. All authors reviewed and approved the final draft.

Funding This study was funded by the China Postdoctoral Science Foundation (funding number: 2019M652589); the Medical Science and Technology Breakthrough Plan Project of Henan province (funding number: SBGJ2018052); the National Natural Science Foundation of China (funding number: U1404814); the Henan Province Science Technology Innovation Talent Support Plan (funding number: 17HASTIT048) and the Science and Technology Department of Henan Province (funding number 134200510018).

Disclaimer The funders had no role in the study design, data collection and analysis, decision to publish, or preparation of the manuscript.

Competing interests No conflict of interest exists in the submission of this manuscript, and the manuscript has been approved by all authors for publication. The work described was original research that has not been published previously and is not under consideration for publication elsewhere, in whole or in part.

Patient and public involvement Patients and/or the public were involved in the design, or conduct, or reporting, or dissemination plans of this research. Refer to the Methods section for further details.

Patient consent for publication Not required.

Ethics approval This study was approved by the Ethics Committee of Zhengzhou University. All participants agreed to join voluntarily and signed the informed consent. Participants could withdraw the study at any time, and they were able to review and amend their transcripts. Anonymity was ensured in all published materials. All the interviews were encrypted and only available to the research team.

\section{Provenance and peer review Not commissioned; externally peer reviewed.}

Data availability statement Data are available upon reasonable request. No additional data are available. The material generated and analysed during the current study cannot be made publicly available for reasons of privacy and confidentiality. However, access to de-identified data sets can be made available on request to Professor Zhang ZX (zhangzx6666@126.com).

Open access This is an open access article distributed in accordance with the Creative Commons Attribution Non Commercial (CC BY-NC 4.0) license, which permits others to distribute, remix, adapt, build upon this work non-commercially, and license their derivative works on different terms, provided the original work is properly cited, appropriate credit is given, any changes made indicated, and the use is non-commercial. See: http://creativecommons.org/licenses/by-nc/4.0/.

\section{ORCID iDs}

Yong-Xia Mei http://orcid.org/0000-0003-4269-3231

Beilei Lin http://orcid.org/0000-0002-6502-7402

\section{REFERENCES}

1 Wang W, Wang D, Liu H, et al. Trend of declining stroke mortality in China: reasons and analysis. Stroke Vasc Neurol 2017;2:132-9.

2 Wang L, Liu J, Yang Y, et al. The prevention and treatment of stroke still face huge challenges-brief report on stroke prevention and treatment in China, 2018. Chinese Cir J 2019;34:105-19.

3 Oliva-Moreno J, Peña-Longobardo LM, Mar J, et al. Determinants of informal care, burden, and risk of burnout in caregivers of stroke survivors: the CONOCES study. Stroke 2018;49:117-7575.

4 Koyanagi A, DeVylder JE, Stubbs B, et al. Depression, sleep problems, and perceived stress among informal caregivers in 58 low, middle-, and high-income countries: a cross-sectional analysis of community-based surveys. J Psychiatr Res 2018;96:115-23.

5 Loh AZ, Tan JS, Zhang MW, et al. The global prevalence of anxiety and depressive symptoms among caregivers of stroke survivors. $J$ Am Med Dir Assoc 2017;18:S869955636.

6 Xie H, Cheng C, Tao Y, et al. Quality of life in Chinese family caregivers for elderly people with chronic diseases. Health Qual Life Outcomes 2016;14:99.

$7 \mathrm{Xi} \mathrm{J}$. The report by president $\mathrm{XI}$ at 19th national Congress of the communist Party of China 2017, 2019. Available: http://cpc.people. com.cn/n1/2017/1028/c64094-29613660.html

8 Han Y, Liu Y, Zhang X, et al. Chinese family caregivers of stroke survivors: determinants of caregiving burden within the first six months. J Clin Nurs 2017;26:4558-66.

9 Liu Z, Albanese E, Li S, et al. Chronic disease prevalence and care among the elderly in urban and rural Beijing, China - a 10/66 Dementia Research Group cross-sectional survey. BMC Public Health 2009;9:1-11.

10 Rubbens E, Clerck LD, Swinnen E. Effectiveness of interventions to decrease the physical and mental burden and strain of informal caregivers of stroke patients: a systematic review. In: Converging clinical and engineering research on neurorehabilitation II. Springer, Cham, 2017: 299-303. ISBN: 978-3-319-46669-9.

11 Pucciarelli G, Vellone E, Savini S, et al. Roles of changing physical function and caregiver burden on quality of life in stroke: a longitudinal dyadic analysis. Stroke 2017;48:733.

12 Lou S, Carstensen K, Jørgensen CR, et al. Stroke patients' and informal carers' experiences with life after stroke: an overview of qualitative systematic reviews. Disabil Rehabil 2017;39:301-13.

13 Grant JS, Clay OJ, Keltner NL, et al. Does caregiver well-being predict stroke survivor depressive symptoms? A mediation analysis. Top Stroke Rehabil 2013;20:44-51.

14 El Masry Y, Mullan B, Hackett M. Psychosocial experiences and needs of Australian caregivers of people with stroke: prognosis messages, caregiver resilience, and relationships. Top Stroke Rehabil 2013;20:356-68.

15 Barbic SP, Mayo NE, White CL, et al. Emotional vitality in family caregivers: content validation of a theoretical framework. Qual Life Res 2014;23:2865-72.

16 Bower JE, Moskowitz JT, Epel E. Is benefit finding good for your health? pathways linking positive life changes after stress and physical health outcomes. Curr Dir Psychol Sci 2009;18:337-41.

17 Cavell S, Broadbent E, Donkin L, et al. Observations of benefit finding in head and neck cancer patients. Eur Arch Otorhinolaryngol 2016;273:1-7.

18 Cassidy T, McLaughlin M, Giles M. Benefit finding in response to general life stress: measurement and correlates. Health Psychol Behav Med 2014;2:268-82.

19 Mei Y, Wilson S, Lin B, et al. Benefit finding for Chinese family caregivers of community-dwelling stroke survivors: a cross-sectional study. J Clin Nurs 2018;27:e1419-28.

20 Lim KK, Chong WH. Moderating effect of child's autism spectrum disorder (ASD) diagnosis on benefit finding and negative affect of parents. Am J Orthopsychiatry 2017;87:357-64.

21 Mock S, Boerner K. Sense making and benefit finding among patients with amyotrophic lateral sclerosis and their primary caregivers. J Health Psychol 2010;15:115-21.

22 Yu DSF, Cheng S-T, Wang J. Unravelling positive aspects of caregiving in dementia: an integrative review of research literature. Int J Nurs Stud 2018;79:1-26. 
23 Male DA, Fergus KD, Stephen JE. The continuous confrontation of caregiving as described in real-time online group chat. J Palliat Care 2015;31:36-43.

24 Haley WE, Allen JY, Grant JS, et al. Problems and benefits reported by stroke family caregivers: results from a prospective epidemiological study. Stroke 2009;40:2129-33.

25 Bacon E, Milne DL, Sheikh Al, et al. Positive experiences in caregivers: an exploratory case series. Behav Cogn Psychother 2009;37:95-114.

26 Yi W. Making the traditional culture as a platform to promote the culture of filial piety. Teacher 2017;2:14-15.

27 Yuan X. Study on the positive experience of the adult children caring for their old parents. Population \& Development 2009;15:65-9.

28 Qiu X, Sit JWH, Koo FK. The influence of Chinese culture on family caregivers of stroke survivors: a qualitative study. J Clin Nurs 2018;27:e309-19.

29 Mackenzie A, Greenwood N, Ann M, Nan G. Positive experiences of caregiving in stroke: a systematic review. Disabil Rehabil 2012;34:1413-22.

30 O'Brien BC, Harris IB, Beckman TJ, et al. Standards for reporting qualitative research: a synthesis of recommendations. Acad Med 2014;89:1245-51.

31 Lin Y, Li P, Liu S. Assessment of ability of daily life of stroke patients. Chi J Clin Reh 2002;6:1249-51.

32 Trotter RT. Qualitative research sample design and sample size: resolving and unresolved issues and inferential imperatives. Prev Med 2012;55:398-400.

33 Braun V, Clarke V. Using thematic analysis in psychology. Qual Res Psychol 2006;3:77-101.

34 Santos HPO, Black AM, Sandelowski M. Timing of translation in Cross-Language qualitative research. Qual Health Res 2015;25:134-44.
35 Graneheim UH, Lindgren B-M, Lundman B. Methodological challenges in qualitative content analysis: a discussion paper. Nurse Educ Today 2017;56:29-34.

36 Seow D, Netto N, Goh J, et al. P3-475: growing and gaining through caregiving in dementia. Alzheimer's \& Dementia 2008;4:T661.

37 Peacock S, Forbes D, Markle-Reid M, et al. The positive aspects of the caregiving journey with dementia: using a strengths-based perspective to reveal opportunities. J Appl Gerontol 2010;29:640-59.

38 Parveen S, Morrison V. Predicting caregiver gains: a longitudinal study. Br J Health Psychol 2012;17:711-23.

39 Carbonneau H, Caron C, Desrosiers J. Development of a conceptual framework of positive aspects of caregiving in dementia. Dementia 2010;9:327-53.

40 Cheng S-T, Mak EPM, Lau RWL, et al. Voices of Alzheimer caregivers on positive aspects of caregiving. Gerontologist 2016;56:u118:451-60.

41 Ribeiro O, Paúl C. Older male carers and the positive aspects of care. Ageing Soc 2008;28:165-83.

42 Jervis LL, Boland ME, Fickenscher A. American Indian family caregivers' experiences with helping elders. J Cross Cult Gerontol 2010;25:355-69.

43 Ekstam L, Johansson U, Guidetti S, et al. The combined perceptions of people with stroke and their carers regarding rehabilitation needs year after stroke: a mixed methods study. BMJ Open 2015;5:e6784.

44 Bakas T, McCarthy M, Miller ET. Update on the state of the evidence for stroke family caregiver and dyad interventions. Stroke 2017;48:e122.

45 Pillemer K, Suitor JJ. Peer support for Alzheimer's caregivers: is it enough to make a difference? Res Aging 2002;24:171-92.

46 Lee RLT, Mok ESB. Seeking harmony in the provision of care to the stroke-impaired: views of Chinese family caregivers. J Clin Nurs 2011;20:1436-44. 